\title{
The Effect of Different Forms of Synaptic Plasticity on Pattern Recognition in the Cerebellar Cortex
}

\author{
Giseli de Sousa, Rod Adams, Neil Davey, Reinoud Maex, Volker Steuber \\ Science and Technology Research Institute, \\ University of Hertfordshire, Hatfield, Herts, UK \\ \{G.Sousa, R.G.Adams, N.Davey, R.Maex1,V.Steuber\}@herts.ac.uk
}

\begin{abstract}
Many cerebellar learning theories assume that long-term depression (LTD) of synapses between parallel fibres (PFs) and Purkinje cells (PCs) provides the basis for pattern recognition in the cerebellum. Previous work has suggested that PCs can use a novel neural code based on the duration of silent periods. These simulations have used a simplified learning rule, where the synaptic conductance was halved each time a pattern was learned. However, experimental studies in cerebellar slices show that the synaptic conductance saturates and is rarely reduced to less than $50 \%$ of its baseline value. Moreover, the previous simulations did not include plasticity of the synapses between inhibitory interneurons and PCs. Here we study the effect of LTD saturation and inhibitory synaptic plasticity on pattern recognition in a complex PC model. We find that the PC model is very sensitive to the value at which LTD saturates, but is unaffected by inhibitory synaptic plasticity.
\end{abstract}

Keywords: Associative memory, Long-term depression, Purkinje cell, Cerebellum

\section{Introduction}

The cerebellum is a part of the brain involved in a multitude of tasks, including motor control, and its functioning is responsible for the smoothness and precision of movements. These skills are improved by a process called motor learning, which is often assumed to be implemented by a form of synaptic plasticity known as long-term depression (LTD). LTD is a long-lasting decrease in synaptic strength due to a loss of AMPA receptors in the postsynaptic membrane [1]. In the cerebellum, LTD has been shown to occur at the synapses between Purkinje cells (PCs) and their excitatory inputs: climbing fibres (CFs) and parallel fibres (PFs). More specifically, cerebellar LTD is an associative process in which the strength of a PF synapse onto a PC is depressed when the CF and PF are activated at the same time. 
Classical cerebellar learning theories suggest that a PC can learn to discriminate between different activity patterns presented by its thousands of afferent PFs, due to LTD of the PF synapses [2]. It is assumed that as a result of LTD, the PC firing rate will be reduced when a learned pattern is presented again, and the PC will exert less inhibition on the deep cerebellar nuclei (Fig. 1). As a consequence, the cerebellar output should be increased, which could implement motor learning [1, 3].

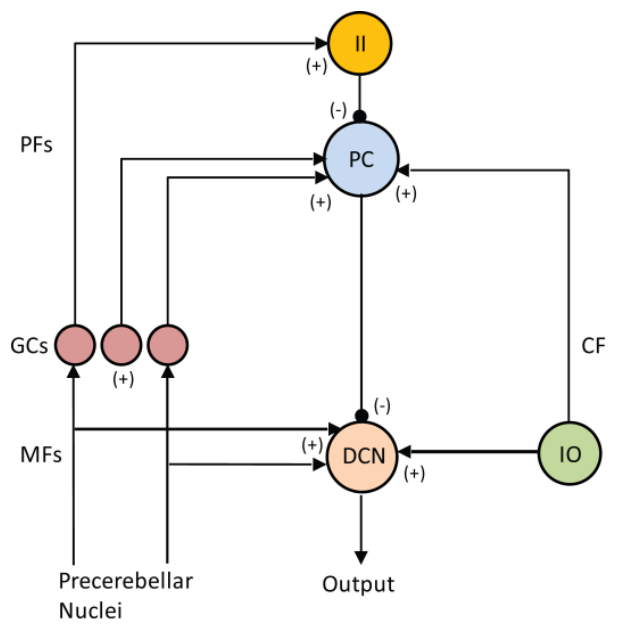

Fig. 1. Schematic diagram of the cerebellar circuitry. Purkinje cells $(P C s)$ receive excitatory inputs $(+)$ from $\sim 150,000$ parallel fibres $(P F s)$ and a single climbing fibre $(C F)$, and inhibitory inputs (-) from inhibitory interneurons $(I I)$, and in turn inhibit the deep cerebellar nuclei $(D C N)$. Also shown are: mossy fibres $(M F s)$, granule cells $(G C s)$ and the inferior olive $(I O)$.

Recent work on cerebellar pattern recognition has demonstrated that this view is too simple. A combined theoretical and experimental study suggested that PCs can use a novel neural code based on the duration of their silent periods, where shorter pauses are produced in response to learned patterns [4] (Fig. 2A). This form of neural coding diverges from the classical view that uses the number or timing of individual spikes to distinguish between novel and learned patterns. In the computer simulations and experiments, the pause was compared with other spike response features like the number of spikes in a fixed time window after pattern presentation and the latency of the first spike in the response, and it was shown that the length of the pause was the best criterion for cerebellar PCs to identify learned patterns (Fig. 2B).

The previous simulations (see Methods) applied a simplified learning rule, where the AMPA receptor conductance was decreased by $50 \%$ each time a pattern was learned. After having stored a number of PF patterns, this could result in very small AMPA receptor conductances. However, experiments with LTD induction in cerebellar slices hardly ever result in mean AMPA receptor conductances of less than $50 \%$ of the pre-induction baseline $[5,6]$. We have therefore investigated a different learning rule with AMPA receptor conductances that saturate at varying values and have studied the effect of this learning rule in pattern recognition simulations. 
A

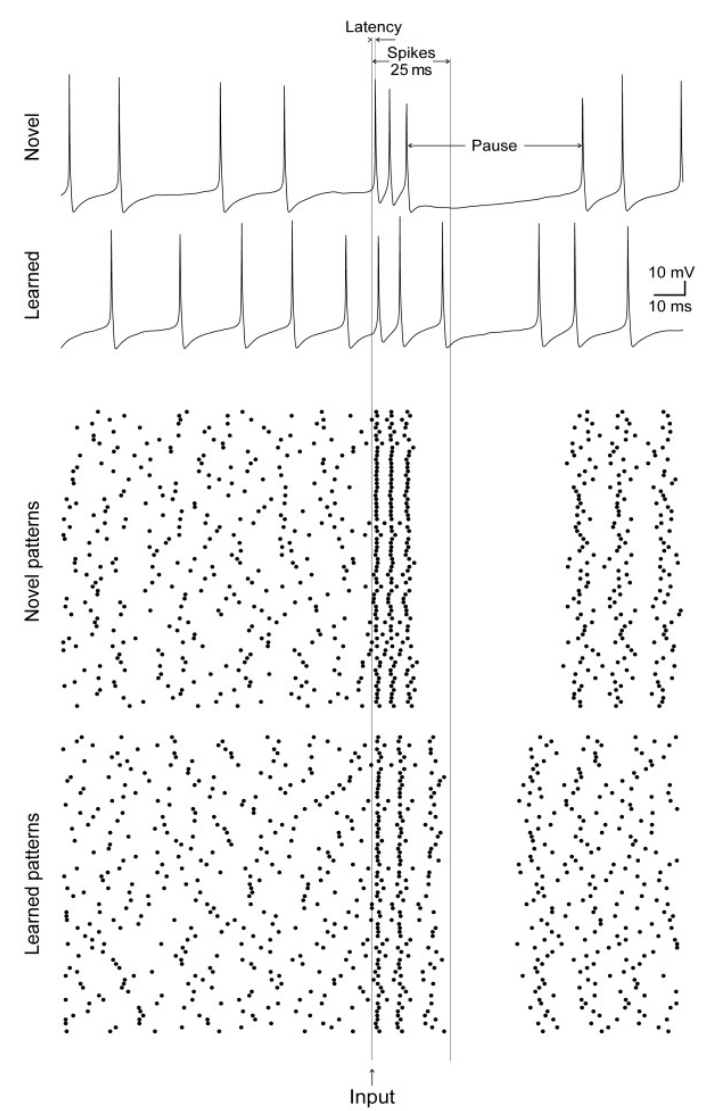

B
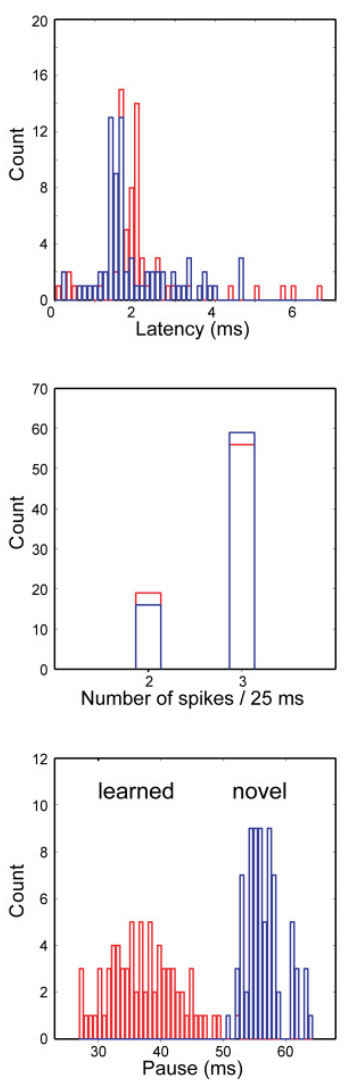

Fig. 2. Responses of a model Purkinje cell to novel and learned patterns of PF input. $(A)$ Upper: The pause evoked by a novel pattern is longer than that for a learned pattern. Lower: Raster plot showing the responses to 75 learned and 75 novel patterns. $(B)$ Response distribution for three different spike features. Upper: Latency of first spike after pattern presentation. Middle: Number of spikes in the first $25 \mathrm{~ms}$. Lower: Length of pause (from [4]).

Another contribution of the present work is to study the effect of LTD at the inhibitory synapses made by interneurons onto PCs (Fig. 1). It has recently been described that this inhibitory synaptic plasticity results in a mean depression of inhibitory inputs down to $75 \%$ of their original values [5]. We have run computer simulations to investigate the effect of different amounts of inhibitory synaptic plasticity on pattern recognition. 


\section{Methods}

\section{Purkinje Cell Model}

The simulations were performed using the GENESIS neural simulator [7], with additional routines implemented in $\mathrm{C}++$ and MATLAB. We simulated a multicompartmental PC model with active dendrites and soma, as described in detail in references $[8,9]$. The model morphology was based on a reconstruction of a guineapig Purkinje cell [10]. Ten different types of voltage-dependent channels were modelled using Hodgkin-Huxley-like equations. The soma compartment had a fast and persistent $\mathrm{Na}^{+}$conductance, a delayed rectifier, a transient A-type $\mathrm{K}^{+}$ conductance, a non-inactivating $\mathrm{M}$-type $\mathrm{K}^{+}$conductance, an anomalous rectifier and a low-threshold T-type $\mathrm{Ca}^{2+}$ conductance. The dendritic compartments contained a Purkinje-cell specific high-threshold P-type and a low-threshold T-type $\mathrm{Ca}^{2+}$ conductance, two different types of $\mathrm{Ca}^{2+}$-activated $\mathrm{K}^{+}(\mathrm{KCa})$ conductances and an $\mathrm{M}$ type $\mathrm{K}^{+}$conductance. Each cell was originally modelled with 147,400 dendritic spines, which were activated randomly by a sequence of PF inputs at an average frequency of $0.28 \mathrm{~Hz}$. The background excitation was balanced by tonic inhibition, which made the model fire simple spikes at an average frequency of $48 \mathrm{~Hz}$. Due to the large number of dendritic spines, which made the simulations computationally expensive, a simplified version of the model was constructed by decreasing the number of spines to $1 \%$ of the original number. To compensate for this reduction, the rate of PF excitation was increased to an average frequency of $28 \mathrm{~Hz}$. As this simplified model gave identical results as the full model, it was used in the simulations presented here.

To study the effect of plasticity at inhibitory synapses, the model was provided with feed-forward inhibitory input by activating a variable number of inhibitory synapses onto the soma and main dendrite. The inhibitory input followed the synchronous activation of excitatory PFs synapses with a delay of $1.4 \mathrm{~ms}$. Inhibition/excitation ratios were measured as ratios of the mean inhibitory postsynaptic current (IPSC) peak to the mean excitatory postsynaptic current (EPSC) peak when the model was voltage clamped to $-40 \mathrm{mV}$.

\section{Pattern Recognition}

The pattern recognition simulations were performed in two steps. First, a number of random binary input patterns were generated, initially 200, and half of these patterns were learned by a corresponding artificial neural network (ANN). The ANN used was a modified version of an associative net with feed-forward connections between its inputs and output [11] and was trained by applying a modified version of the LTD learning rule [12] (see below). The simulations of the ANN consisted of two phases: learning and recall.

In the learning phase, the weights of all synapses that received a positive input during the presentation of a pattern were set to a constant value. This LTD saturation value was kept constant and unaffected by further pattern presentations, different 
from the learning rule that had been used in the previous simulations [4]. During the recall phase, the response of the ANN was given by the sum of the weights of all synapses that were associated with active inputs, which resulted in responses of the ANN to stored patterns that were lower than those to novel patterns (Fig. 3).

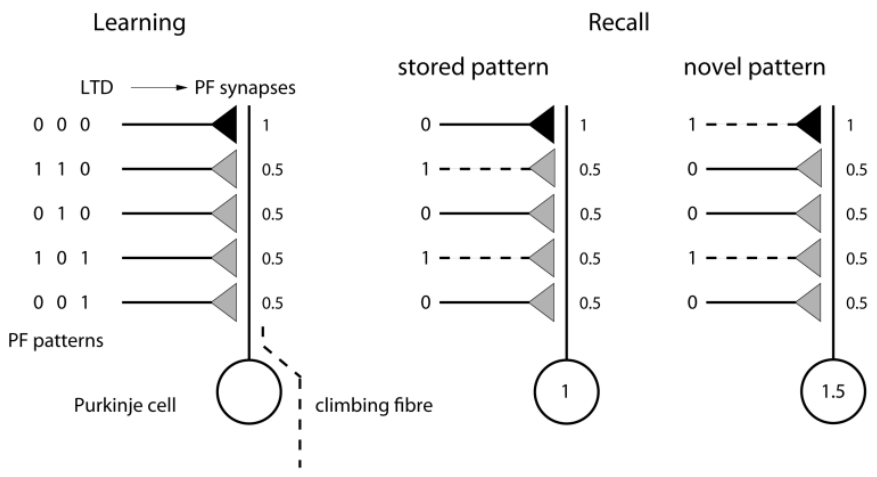

Fig. 3. Simplified schematic of the ANN model. Left side: during learning, three example PF patterns are stored by changing the synaptic weights that are associated with active input lines from their initial value of 1 to an LTD saturation value of 0.5 (this value is varied between different simulations). Right side: during recall, the responses to a stored and a novel pattern are calculated as dot product of input vector and weight vector, resulting in values of 1 and 1.5, respectively (note the difference to the original diagram in [12]).

In the second phase of the pattern recognition simulations, the vector of synaptic weights was transferred from the ANN onto AMPA receptor conductances in the multi-compartmental PC model. This represents learning the PF patterns by depressing the corresponding AMPA receptor conductances during LTD induction. To test the recall of learned patterns, the PC model was then presented with a corresponding pattern of synchronous AMPA receptor activation at the PF synapses.

The discrimination between novel and learned pattern in the two models was evaluated by calculating a signal-to-noise ratio $[13,14]$ :

$$
s / n=\frac{\left(\mu_{s}-\mu_{n}\right)^{2}}{0.5\left(\sigma_{s}^{2}+\sigma_{n}^{2}\right)}
$$

where $\mu_{\mathrm{s}}$ and $\mu_{\mathrm{n}}$ represent the mean values and $\sigma_{\mathrm{s}}{ }^{2}$ and $\sigma_{\mathrm{n}}{ }^{2}$ represent the variances of the responses to stored and novel patterns, respectively. In the PC model, three different features of the spike response were tested as criteria to distinguish stored from novel patterns: the latency of the first spike fired after pattern presentation, the number of spikes in a $25 \mathrm{~ms}$ time window after pattern presentation, and the duration of a silent period that followed the pattern presentation (see response distributions for these three different metrics in Fig. 2B). In all cases studied, the pause duration was the best criterion, and only pause based signal-to-noise ratios are presented here. 


\section{Results}

\section{LTD Saturation and the Number of Active PF Inputs}

We initially investigated the effect of varying two parameters that were expected to affect the pattern recognition performance: the value at which LTD saturated and the number of active PFs for each pattern.
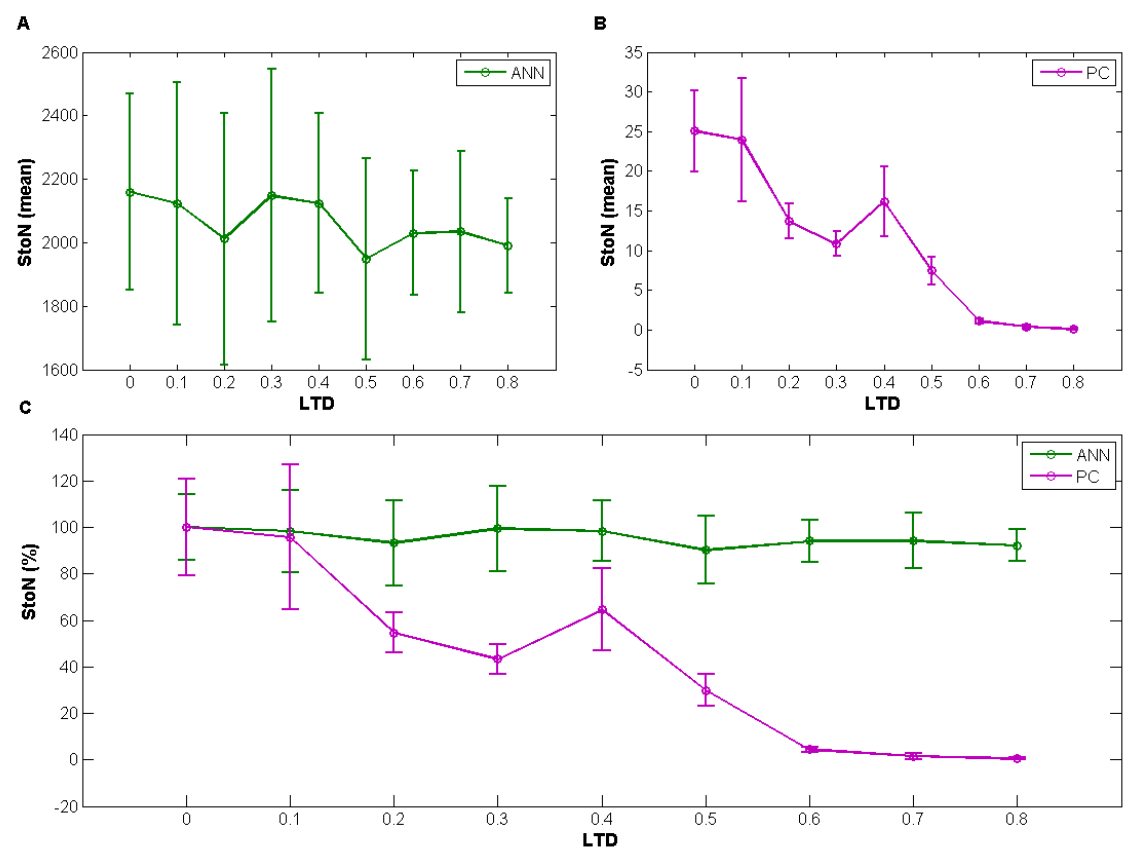

Fig. 4. Pattern recognition performance of the two models for a range of LTD values. The performance was evaluated by calculating $\mathrm{s} / \mathrm{n}$ ratios for the ANN $(A)$ and the PC model $(B)$. The relative decreases in $\mathrm{s} / \mathrm{n}$ ratio are compared in $(C)$, showing that the $\mathrm{PC}$ model is more sensitive to LTD saturation than the ANN. Error bars indicate standard deviation (SD).

To study the effect of LTD saturation, we varied the LTD saturation value over a range from zero to 0.8 , while keeping the same numbers of active PFs (1000) and PF patterns (100 novel and 100 stored) as in previous work [4]. We found that the ANN was insensitive to the amount of LTD induced (Fig. 4A). In contrast, the pattern recognition capacity based on the duration of silent periods in the PC model improved when the LTD saturation value decreased, with an optimal performance when the synaptic weights of active PFs were set to zero (Fig. 4B). The relative sensitivities of the ANN and the PC model to the amount of LTD induced are compared in Fig. 4C. While the ANN was unaffected by varying the amount of LTD, increasing the LTD saturation value to 0.8 in the PC model reduced the signal-to-noise ratio down to $0.4 \pm$ $0.4 \%(n=10)$ of the optimal value obtained by switching off the synapses completely. 
For LTD saturation values below 0.5 , the PC model performed as well as or better than the previous model with a non-saturating learning rule [4].
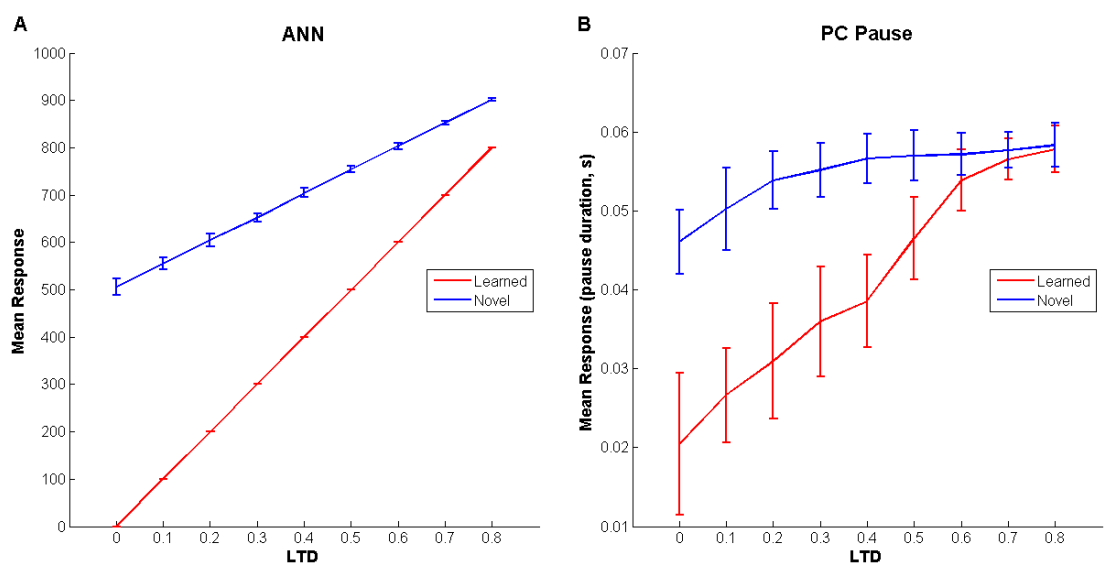

Fig. 5. Relationship between the LTD saturation value and the mean responses to stored and novel patterns in the ANN and the PC model. Although the difference between the mean responses to stored and novel patterns decreases with increasing LTD saturation values in both cases, in the ANN the variance of responses to novel patterns also decreases. This results in $\mathrm{s} / \mathrm{n}$ ratios in the ANN that are independent of the LTD saturation value. Same simulation parameters as in Fig. 4. Error bars indicate SD.

The reason for the difference in sensitivity of the ANN and the PC model to varying amounts of LTD became apparent when the mean responses of the two models to stored and novel patterns were plotted against the LTD saturation value (Fig. 5). In the PC model, increasing LTD saturation values reduced the difference in pause duration between stored and novel patterns, with standard deviations that were affected to a much lesser extent (Fig. 5B). This led to the drastic reduction in $\mathrm{s} / \mathrm{n}$ ratio for weak LTD shown in Figure 4. In the ANN, the difference between the mean responses to stored and novel patterns was affected much less by the LTD saturation value, while the standard deviation of responses to novel patterns decreased with increasing LTD saturation values (Fig. 5A). Based on Equation (1), the constant signal-to-noise ratio of the ANN in the presence of varying amounts of LTD can be explained by a linear relationship between the squared difference of the mean responses to stored and novel patterns $\left(\mu_{\mathrm{s}}-\mu_{\mathrm{n}}\right)^{2}$ and the variance of the responses to novel patterns $\sigma_{\mathrm{n}}^{2}$.

In a second set of simulations, we measured the effect of varying the number of active PFs in each pattern for a range of LTD values. As expected, the performance of the ANN deteriorated for larger numbers of activated PFs, while being independent of the amount of LTD induced over the whole range of numbers of active PFs tested (500-5000, Fig. 6A). In contrast, the PC model showed the best pattern recognition capacity for a range between 1000 and 2000 active PFs and performed consistently worse for higher LTD saturation values (Fig. 6B). 

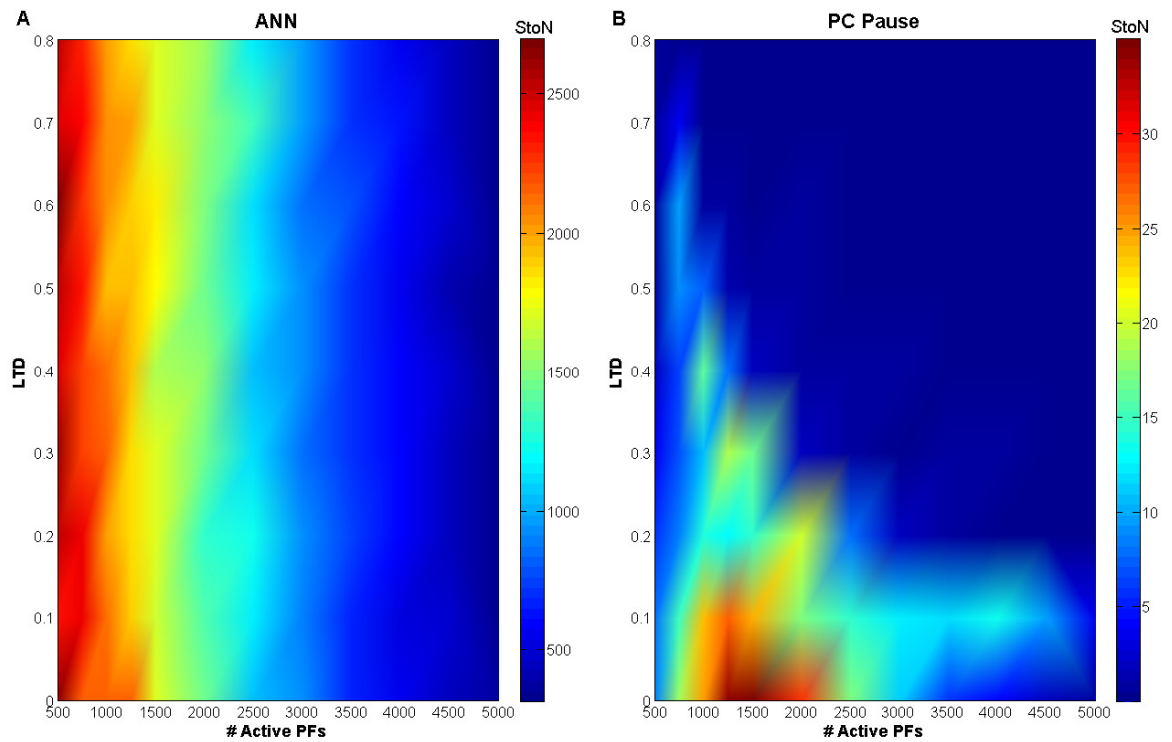

Fig. 6. Pattern recognition performance of the ANN $(A)$ and PC model $(B)$. The colour represents the resulting $\mathrm{s} / \mathrm{n}$ ratio for each combination of a number of active PFs for each pattern (indicated on the x-axis) and an LTD saturation value (y-axis).

\section{Inhibitory Synaptic Plasticity}

To investigate the effect of plasticity at the synapses between inhibitory interneurons and PCs, we initially used an inhibition/excitation ratio of one (see Methods), which is in the range of experimentally observed data from cerebellar slices [5]. We then introduced LTD at the inhibitory synapses and evaluated the pattern recognition performance of the PC for different numbers of patterns. The effect of inhibitory LTD was examined by depressing the inhibitory conductance to values between $25 \%$ and $75 \%$ of their pre-depression baseline. We used four different simulation setups (Fig. 7): no inhibition, plasticity at inhibitory synapse for stored and novel PF patterns, plasticity for stored patterns only and no plasticity for both patterns, that is, maintaining the baseline amplitude value for the original inhibition/excitation ratio [5].

We found that the pattern recognition performance of the PC model was unaffected by the presence of inhibitory LTD, even in the extreme case where the inhibitory plasticity was restricted to learned PFs patterns. 

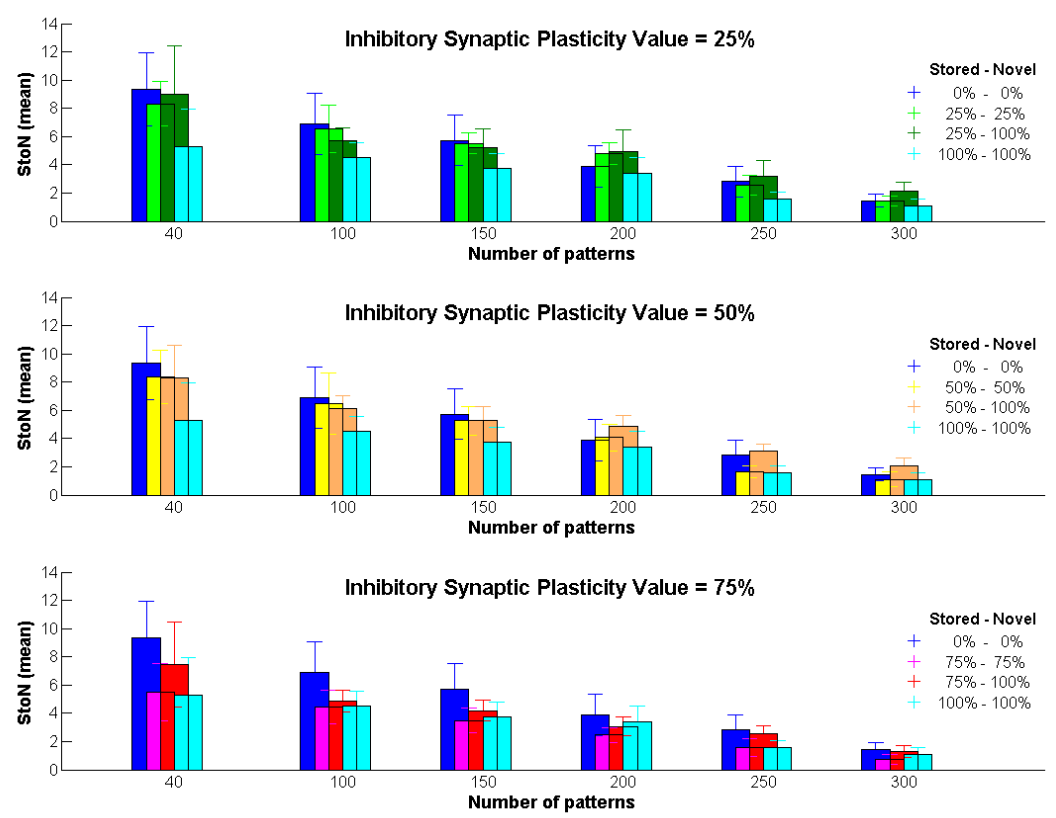

Fig. 7. Depression at inhibitory synapses. Three different inhibitory synaptic plasticity rules were applied for varying numbers of patterns. The first bar of each graph shows the $\mathrm{s} / \mathrm{n}$ ratio when no inhibition is applied for both stored and novel patterns, resulting in the best pattern recognition performance. The others bars represent cases with inhibition present, with from left to right: plasticity for both stored and novel patterns, plasticity for stored patterns only and no plasticity for either type of patterns, using the original inhibitory conductances. Error bars indicate SD.

\section{Conclusion}

Previous computer simulations and experiments in cerebellar slices and awake behaving rats suggested that the cerebellum can use a novel neural code that is based on the duration of silent periods in neuronal activity [4]. These simulations used a complex multi-compartmental model of a cerebellar Purkinje cell that had been tuned to replicate a wide range of behaviours in vitro and in vivo $[8,9]$, but they applied a simplified LTD learning rule, which involved dividing the synaptic weights of active PF inputs by two every time a PF pattern was learned. This could result in very small synaptic weights and does not fit experimental data on LTD induction in cerebellar slices, where the mean AMPA receptor conductances saturate and are hardly ever depressed to less than $50 \%$ of their pre-depression baseline values $[5,6]$. Moreover, the previous simulations did not include the plasticity at synapses between inhibitory interneurons and PCs that has recently been characterised [5].

We have studied the effect of inhibitory synaptic plasticity and saturating LTD in the complex PC model. We found that the ability of the PC model to discriminate 
between learned and novel PF input patterns was unaffected by the presence of inhibitory plasticity for a wide range of parameter values.

However, the pattern recognition performance of the PC model was very sensitive to the value at which LTD saturated. In contrast to a corresponding ANN, which was unaffected by the amount of LTD induced, the performance of the PC model was improved by lower LTD saturation values. The best performance resulted from LTD saturation values of zero, which corresponds to silencing the PF synapses completely. Interestingly, large numbers of silent PF synapses have been observed by monitoring microscopically identified PF-PC connections in cerebellar slices [15]. Our simulation results indicate that the discrepancy between the existence of these silent synapses and the apparent saturation of LTD in induction experiments needs to be resolved to understand the connection between LTD and cerebellar learning.

\section{References}

1. Ito, M.: Cerebellar Long-Term Depression: Characterization, Signal Transduction, and Functional Roles. Physiol. Rev., 81(3):1143-1195, (2001)

2. Marr, D.A.: A theory of cerebellar cortex. Journal of Physiology (London), 202:437-470, (1969)

3. Ito, M.: The cerebellum and neural control. Raven Press, New York (1984)

4. Steuber, V., Mittmann, W., Hoebeek, F.E., Silver, R.A., De Zeeuw, C.I., Häusser, M. and De Schutter, E.: Cerebellar LTD and Pattern Recognition by Purkinje Cells. Neuron, 54(1):121-136, (2007)

5. Mittmann, W. and Hausser, M.: Linking Synaptic Plasticity and Spike Output at Excitatory and Inhibitory Synapses onto Cerebellar Purkinje Cells. J. Neurosci., 27(21):5559-5570, (2007)

6. Wang, S.S.H., Denk, W. and Hausser, M.: Coincidence detection in single dendritic spines mediated by calcium release. Nat Neurosci, 3(12):1266-1273, (2000)

7. Bower, J.M. and Beeman, D.: The book of GENESIS: Exploring Realistic Neural Models with the GEneral NEural SImulation System. (2003)

8. De Schutter, E. and Bower, J.M.: An active membrane model of the cerebellar Purkinje cell. I. Simulation of current clamps in slice. Journal of Neurophysiology, 71(1):375-400, (1994)

9. De Schutter, E. and Bower, J.M.: An active membrane model of the cerebellar Purkinje cell: II. Simulation of synaptic responses. Journal of Neurophysiology, 71(1):401-419, (1994)

10.Rapp, M., Segev, I. and Yarom, Y.: Physiology, morphology and detailed passive models of guinea-pig cerebellar Purkinje cells. Journal of Physiology (London), 474(1):101-118, (1994)

11.Willshaw, D.J., Buneman, O.P. and Longuet-Higgins, H.C.: Non-holographic associative memory. Nature, 222:960-962, (1969)

12.Steuber, V. and De Schutter, E.: Long-term depression and recognition of parallel fibre patterns in a multi-compartmental model of a cerebellar Purkinje cell. Neurocomputing, 38:383-388, (2001)

13.Dayan, P. and Willshaw, D.J.: Optimising synaptic learning rules in linear associative memories. Biol Cybern, 65(4):253-265, (1991)

14.Graham, B.P.: Pattern recognition in a compartmental model of a CA1 pyramidal neuron. Network: Computation in Neural Systems, 12(4):473 - 492, (2001)

15.Isope, P. and Barbour, B.: Properties of unitary granule cell-Purkinje cell synapses in adult rat cerebellar slices. Journal of Neuroscience, 22(22):9668-9678, (2002) 